Technical note

\title{
Evaluation of ionization chamber stability checks using various sources
}

\author{
Autumn E. Walter *, Jon B. Hansen, Larry A. DeWerd \\ 1111 Highland Ave, Department of Medical Physics, School of Medicine and Public Health, University of Wisconsin, Madison, WI 53705, USA
}

\section{A R T I C L E I N F O}

\section{Keywords:}

Ionization chambers

Linear accelerator

Check source

QA

\begin{abstract}
A B S T R A C T
Purpose: It is important to check stability of ionization chambers in between regular calibration cycles. Stability checks can include individual ${ }^{60} \mathrm{Co}$ irradiations, use of a beta-emitting check source, or redundant measurements in megavoltage photon beams. While ${ }^{60} \mathrm{Co}$ irradiators are considered stable, they are rarely found in the clinical setting. Thus, this study seeks to compare the precision and efficiency in monitoring chamber stability using ${ }^{90} \mathrm{Sr}$ check sources and linear accelerator beams which are both commonly found in the clinical setting, and compare these sources to ${ }^{60} \mathrm{Co}$.

Methods: Measurements were made with a ${ }^{90} \mathrm{Sr}$ beta-emitting check source and a 6 MV photon beam using a Constancy Check Phantom with three custom inserts to hold the ionization chambers. A comparison of both methods was performed with an Exradin A28 scanning chamber, Wellhofer IC69 Farmer-type chamber, and Exradin A12 Farmer-type chamber. Chamber stability was evaluated with individual charge readings and charge ratios among the three chambers. Results were compared to measurements taken in ${ }^{60}$ Co with three Farmer-type chambers: the NEL 2571, PTW N30001G, and Exradin A12.

Results: Stability of individual charge reading was found to be within $\pm 1.0 \%$ for ${ }^{90} \mathrm{Sr}$ source measurements and $\pm 0.5 \%$ for external beam measurements, including the ${ }^{60} \mathrm{Co}$ comparison. Additionally, the standard deviation of the mean charge ratios ranged from $0.15 \%$ to $0.40 \%$ for ${ }^{90} \mathrm{Sr}$ measurements and from $0.10 \%$ to $0.30 \%$ for the external beam measurements.

Conclusions: This work provides a comparison of techniques used to assess stability of ionization chambers in order to better inform the clinical physicist.
\end{abstract}

\section{Introduction}

Regulatory bodies, such as the United States Nuclear Regulatory Commission (NRC), and professional bodies, such as the International Commission on Radiation Units and Measurements (ICRU) and the American Association of Physicists in Medicine (AAPM) recommend the calibration of ionization chambers at least once every two years [1-3]. To ensure the chamber's stability between calibrations, regular, independent quality assurance (QA) checks should be performed. The Addendum to the AAPM Task Group 51 Report states that the use of ${ }^{60} \mathrm{Co}$ irradiators is the best method for monitoring chamber stability [4]. However, most of these irradiators have been replaced by linear accelerators in the clinic, making cobalt an unrealistic option for many. Alternatively, sequential chamber checks in beta-emitting check sources, cross calibration of chambers, or redundant comparison with other chambers in a linear accelerator beam can be used to check the chamber's stability [5]. While all are valid methods, the redundant checks can provide an additional time savings when compared to other, sequential methods used.

Beta-emitting ${ }^{90} \mathrm{Sr}$ check sources have historically been used to monitor the stability of ion chambers as originally recommended by IEC 60,731 [6]. These sources are long-lived, easily transported within the clinic, and are generally not affected by outside environments [7]. The independence from the environment was demonstrated by McEwen and Taank in their work on the influence of humidity on chamber measurements, where they found Farmer chambers to agree within $\pm 0.5 \%$ across the whole range of relative humidity [8]. In addition, they found the response of the chamber while using the check source to have standard deviations up to $0.019 \%$ for a given humidity and measurement set with the standard deviation of the mean of measurements to be $0.027 \%$ [8]. However, as presented by Sidhu et al., these check sources are typically associated with a larger variability of $\pm 1 \%$ in comparison to checks performed with ${ }^{60} \mathrm{Co}$ or a medical linear accelerator, due to the angular response of the chamber [7]. Additionally, some chambers may

\footnotetext{
* Corresponding author.

E-mail address: aewalter2@wisc.edu (A.E. Walter).
} 
be too large for the check source housing. There are also a number of regulations to be considered for these types of small, transportable sources. As classified in the US NRC Code of Federal Regulations Title 10 Part 20, these types of sources are considered non-exempt radioactive sources, and they have a specific set of regulations that must be followed [9]. For example, specific training and documentation are required when using these sources. In addition, routine leak tests must be performed within the facility monitored through audits. When moving or shipping sources, regulations from agencies, such as the Department of Transportation and Federal Aviation Administration, must be followed as well [9].

The use of an external beam measurements to evaluate a chamber's stability was first suggested in 1984 [10]. This technique makes use of two or more ionization chambers positioned reproducibly in an external beam and compares the ratio of charge readings against a baseline value. It allows for different chamber types to be compared at the same time, either in-air or in-phantom. A study by Rozenfeld and Jette outlines the advantage of the redundancy method [9]. More than a single chamber is necessary, as there is a lower chance of detecting a faulty chamber if only one chamber is used compared to using two or more chambers [10]. It should be noted that multiple electrometer inputs are needed to match the number of chambers used in the redundancy check. In addition, there are regulations that need to be followed when using a linear accelerator, as outlined in the NRC Code of Federal Regulations Title 10 Part 36 [11].

The purpose of this work is to compare the use of a ${ }^{90} \mathrm{Sr}$ check source and external beam qualities when performing ionization chamber stability checks. The overall achievable precision and efficiency in performing both single chamber and redundant chamber checks using these radiation sources are evaluated to give clinicians information on best practices for their program.

\section{Methods}

An NEL 2571 Farmer-type chamber (QADOS, Berkshire, UK), a PTW N30001G Farmer-type chamber (PTW, Freiburg, Germany), and an Exradin A12 Farmer-type chamber (Standard Imaging, Middleton, WI), were used to make in-air measurements to check air kerma response in Theratron T1000 ${ }^{60} \mathrm{Co}$ irradiator. A MAX4000 electrometer was used, and chambers were aligned using the lasers in the room. An Exradin A12, Exradin A28 scanning chamber (Standard Imaging, Middleton, WI), and Wellhofer IC69 Farmer-type chamber (IBA, Schwarzenbruck, Germany) were used to compare ${ }^{90} \mathrm{Sr}$ and linear accelerator measurements. A MAX 4000 electrometer with a single input channel and a SuperMAX electrometer (Standard Imaging, Middleton, WI) with two input channels calibrated at an ADCL were used to monitor the stability of the aforementioned chambers. A T48012 ${ }^{90} \mathrm{Sr}$ check source (PTW, Freiburg, Germany) with an opening at the top to insert each chamber was used for check source measurements. For external beam measurements, a $30 \times 30 \times 30 \mathrm{~cm}^{3}$ Virtual Water ${ }^{\mathrm{TM}}$, Constancy Check Phantom manufactured by Standard Imaging (Middleton, WI) was used. The phantom had three holes spaced $3 \mathrm{~cm}$ apart, center-to-center, with custom inserts for each chamber type. Additionally, blank plugs were used to fill any holes in the phantom without a chamber. This phantom requires three electrometer inputs for all three ionization chambers to be read at the same time. The chambers were centered at a depth of $6.5 \mathrm{~cm}$ with $6.5 \mathrm{~cm}$ of backscattering material using additional Virtual Water ${ }^{\mathrm{TM}}$ slabs. External beam measurements were performed on a Varian 21EX Clinac ${ }^{\circledR}$ (Palo Alto, California) at the UWMRRC with a 6 MV beam at a dose rate of $600 \mathrm{MU} / \mathrm{min}$.

\section{Measurements}

\section{1. ${ }^{60}$ Co measurements}

NIST calibrated chambers are intercompared on at least a yearly

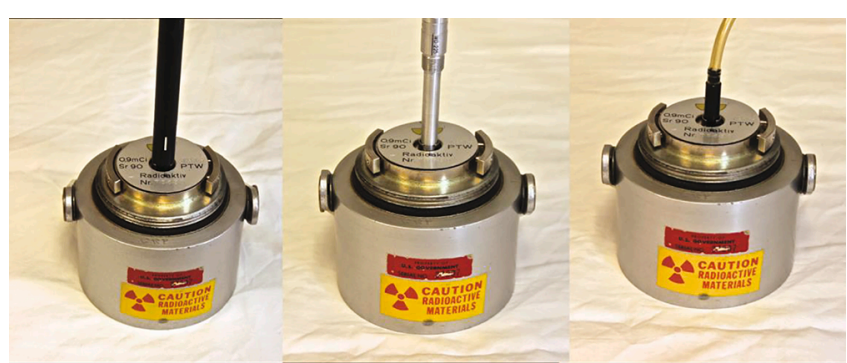

Fig. 1. Chamber placement in the ${ }^{90} \mathrm{Sr}$ check source for the A12 chamber (left), IC69 chamber (center), and A28 chamber (right). Note that the A28 and IC69 chambers were inserted with the tip of the thimble resting on the bottom of the cavity, while the wider stem of the A12 chamber did not allow for the chamber to be inserted past the thimble.

basis using the UWADCL ${ }^{60} \mathrm{Co}$ source. The three chambers used were previously calibrated at NIST. The comparison was done using the Exradin A12 chamber as the reference value. Measurements were taken in air and were corrected for temperature, pressure, and decay of the source. The percent difference of the NEL 2571 and PTW N30001G from the A12 were tracked over a period of 10 years. These measurements were performed in order to compare the previous gold standard for constancy checks with two more clinically feasible methods.

\section{2. ${ }^{90} \mathrm{Sr}$ measurements}

The A28 chamber was used to investigate the response profile based on its placement from the check source. The distance of the thimble tip to the bottom of the check source cavity was adjusted in $1 \mathrm{~mm}$ increments. Fifteen-second charge readings were taken at each depth with $\mathrm{a}+300 \mathrm{~V}$ bias applied. Only the A28 was used for this investigation, as it is a smaller volume, scanning chamber, giving a more precise assessment of the location of the hot spot of the beta source. Rotational dependence was also evaluated for each chamber in the check source at $90^{\circ}$ increments. Each chamber's rotational orientation was preserved by aligning the line on the stem of each chamber with a mark on the front of the check source, denoted as the 0 location. All charge readings were repetitive, with chamber reinserted before each measurement.

Following the initial assessment of the set-up uncertainties, each chamber was inserted into the ${ }^{90} \mathrm{Sr}$ check source to a specified depth, aligned at the 0 position to perform stability checks. As shown in Fig. 1, the A28 and IC69 chambers were placed with the tip of the thimble resting on the floor of the check source cavity. However, only the thimble of the A12 chamber was placed in the check source, as the diameter of the stem of the chamber was too large for the opening on the check source. This chamber was still included in this work to provide a complete comparison between the linear accelerator and ${ }^{90} \mathrm{Sr}$ methods, as the A12 is a reference ionization chamber. The use of this chamber also highlights a limitation of the ${ }^{90} \mathrm{Sr}$ check source.

Each chamber had a $+300 \mathrm{~V}$ bias applied to collect negative charge using the MAX4000 electrometer, and the current was allowed to stabilize upon insertion. Repetitive, $15 \mathrm{~s}$ charge readings were taken after each reinsertion of the chamber into the check source cavity. Ten readings per trial were taken for each chamber. Temperature inside of the source cavity and pressure within the room were recorded before and after each charge measurement. To monitor chamber stability, individual charge readings and ratios between chamber pairs were tracked over time, with a minimum of three days between trials. The ${ }^{90} \mathrm{Sr}$ source decay was accounted for using the 29-year half-life.

The achievable precision of using single chamber measurements in ${ }^{90} \mathrm{Sr}$ to monitor stability was evaluated by calculating the average charge reading for a given trial with,

$M_{\text {corr }}^{\mathrm{x}}=M_{\mathrm{raw}}^{\mathrm{x}} P_{\text {elec }}^{\mathrm{x}} P_{\mathrm{TP}}^{\mathrm{x}} P_{\text {decay }}$ 


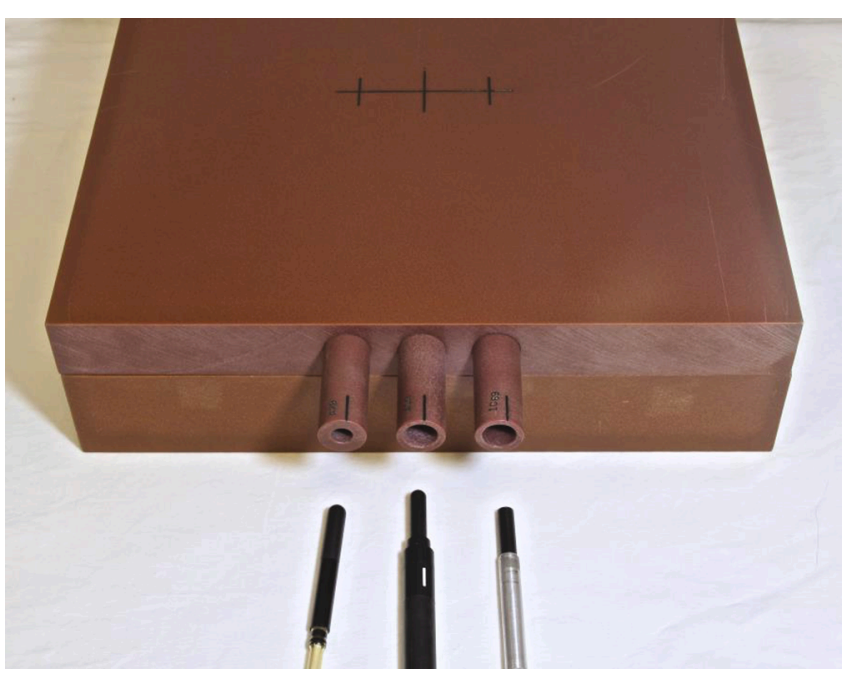

Fig. 2. Constancy Check Phantom (Standard Imaging, Middleton, WI) with chamber inserted and backscattering material. A $5 \mathrm{~cm}$ slab of Virtual Water ${ }^{\mathrm{TM}}$ was also added on top of the phantom shown for all measurements. The chamber location in front of the phantom indicates its location throughout all chamber stability trials.

where $M_{\text {raw }}^{\mathrm{x}}$ is the average uncorrected charge reading, $P_{\text {elec }}^{\mathrm{x}}$ is the electrometer calibration coefficient, and $P_{\mathrm{TP}}^{\mathrm{x}}$ is the temperature and pressure correction factor using chamber model $\mathrm{x}$ for a given measurement trial. The factor $P_{\text {decay }}$ accounts for the decay of the ${ }^{90} \mathrm{Sr}$ check source with the 28-year half-life. The average of the pre- and post-irradiation temperatures and pressures for each chamber was used to determine $P_{\mathrm{TP}}^{\mathrm{x}}$ and applied to a given chamber's readings. In addition to the monitoring individual chamber readings within the ${ }^{90} \mathrm{Sr}$ check source, ratios of charge readings between chamber pairs were determined for each trial using,

$R_{x^{\prime}}^{x}=\frac{M_{\mathrm{corr}}^{x}}{M_{\mathrm{corr}}^{x^{x}}}$

where $M_{\text {corr }}^{x}$ is the average corrected charge reading for chamber model x and $M_{\text {corr }}^{x^{\prime}}$ is the average corrected charge reading for the chamber $\mathrm{x}$ ' for a given ratio. While this method is not truly a redundant chamber check, as all three chambers were not used simultaneously, ratios were still taken to provide an adequate comparison to the linear accelerator methods.

\subsection{Linear accelerator measurements}

Linear accelerator measurements were performed with the Constancy Check Phantom. The A12 chamber was placed in the center hole of the phantom with the A28 chamber to its left and IC69 chamber to its right, as shown in Fig. 2. The phantom was placed at a source-to-surface distance (SSD) of $100 \mathrm{~cm}$, and measurements were carried out at a field size of $20 \times 20 \mathrm{~cm}^{2}$. All electrometers were used in continuous mode, and the current was allowed to stabilize before the beam was turned on to avoid any leakage current. Similar results were obtained using a $10 \times 10 \mathrm{~cm}^{2}$ field. The A12 chamber was connected to the MAX4000 electrometer while the A28 and IC69 were connected to the two channels of the SuperMAX. Ten, $100 \mathrm{MU}$ irradiations were performed for each field size with a potential of $+300 \mathrm{~V}$ applied to each chamber to collect negative charge. Temperature within the phantom inserts and room pressure were recorded before the initial irradiation and after the final irradiation to determine the atmospheric correction factor, $P_{\mathrm{TP}}$. The atmospheric correction factor as well as an electrometer correction factor were applied to correct these charge readings. Eq. (2) was also

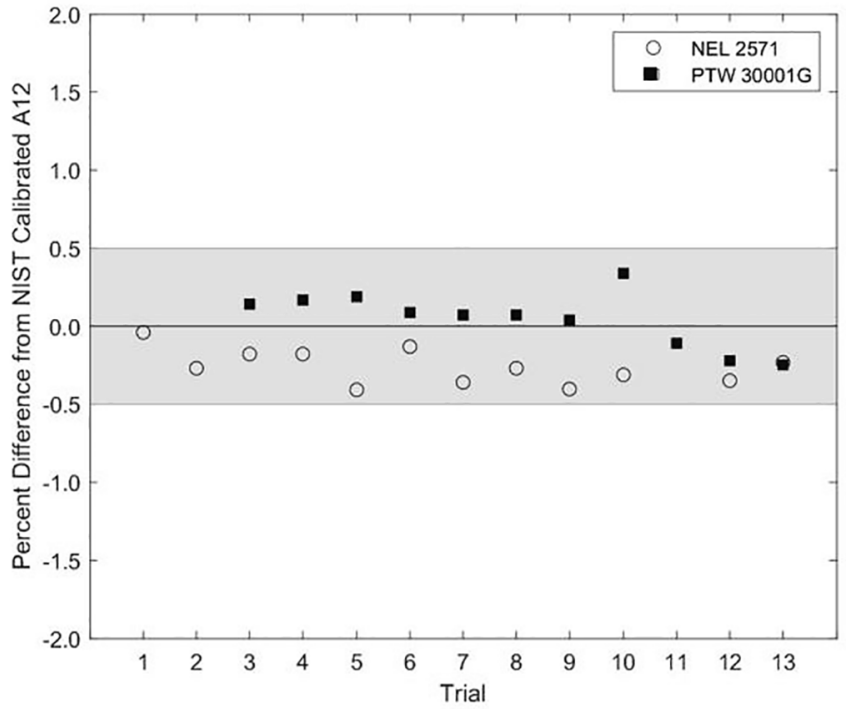

Fig. 3. Comparison of NEL 2571 and PTW N30001G NIST calibrations with Exradin A12 NIST calibration in ${ }^{60} \mathrm{Co}$.

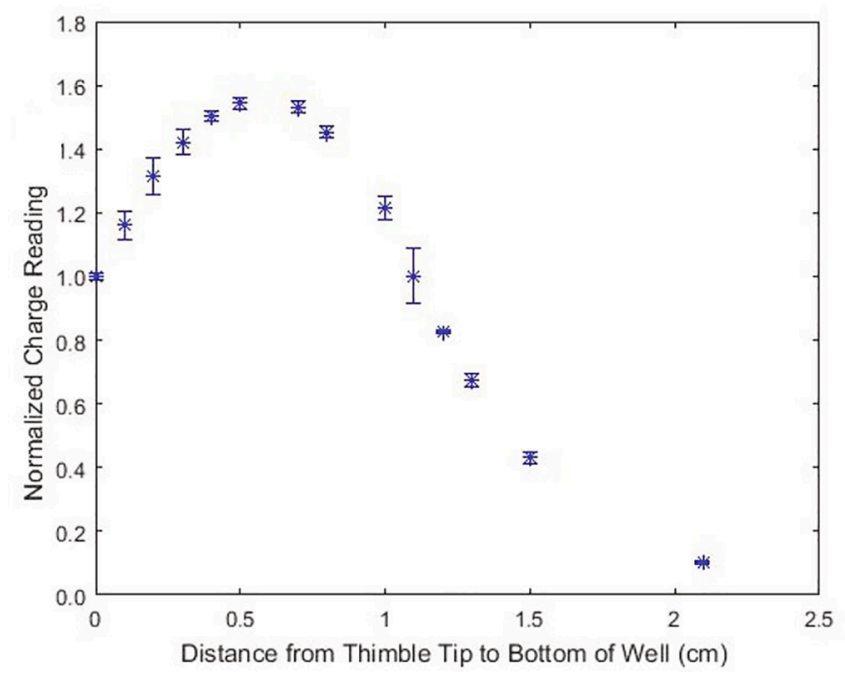

Fig. 4. Scanning data for the A28 chamber in the ${ }^{90} \mathrm{Sr}$ check source as a function of the distance from the chamber thimble tip to the bottom of the cavity. The error bars represent the standard deviation of charge readings at that depth.

used to calculate the charge ratios for the linear accelerator. Both individual charge readings and charge ratios were tracked over time to monitor chamber stability with a minimum of three days between trials. The phantom was aligned using lasers and markings on the accelerator couch for each trial, and the SSD was set with a pointer for accurate placement.

\section{Results}

\section{1. ${ }^{60}$ Co measurements}

Fig. 3 shows a comparison of the NEL 2571 and PTW N30001G chambers compared to the A12 chamber over the 10-year period. All values were within $\pm 0.5 \%$, with a maximum variation of approximately $0.4 \%$. No clear trend was observed over the time period of measurements. 


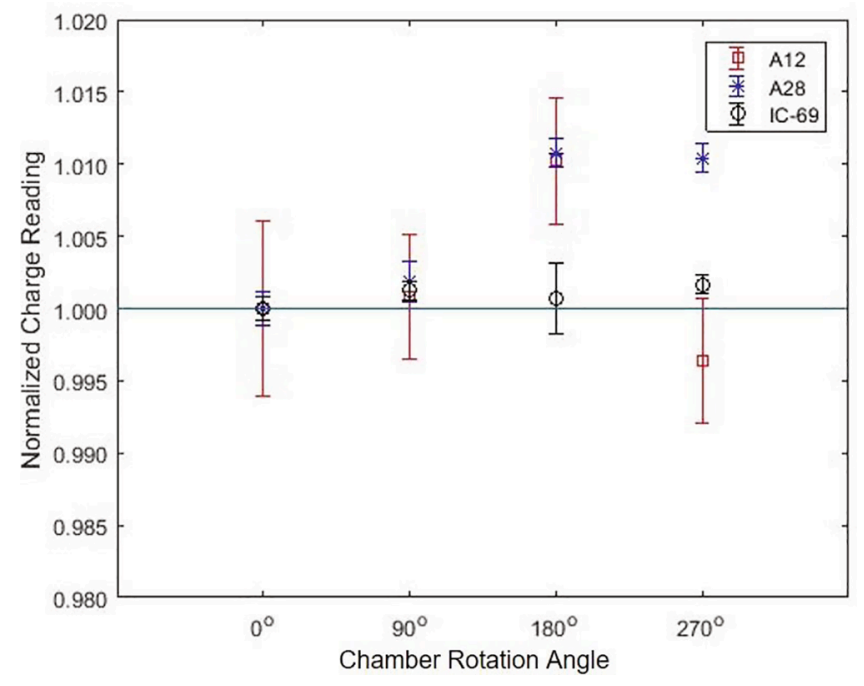

Fig. 5. Rotational variation for the A12, A28, and IC69 chambers in the ${ }^{90} \mathrm{Sr}$ check source. Angles indicate the orientation of the chamber relative to the marker line on the front of the check source, denoted as 0 . Error bars represent the standard deviation of the measurements at a given angle.

\section{2. ${ }^{90} \mathrm{Sr}$ measurements}

\subsubsection{Depth and rotational dependence}

Fig. 4 shows the results from a scan of the A28 chamber response within the ${ }^{90} \mathrm{Sr}$ check source cavity and illustrates that there is a point of maximum exposure near $0.5 \mathrm{~cm}$ in the check source. The error bars represent one standard deviation of repeated charge measurements at a given point during the scan without reinserting the chamber. Additionally, chamber response varied by up to $\pm 1 \%$ when rotated about the Oposition of the check source, as shown in Fig. 5.

\subsubsection{Chamber stability using ${ }^{90} \mathrm{Sr}$}

For each trial, the total time to set up and acquire ten measurements with all three chambers was approximately $38 \mathrm{~min}$. Fig. 6 shows the average chamber readings for multiple trials normalized to the initial baseline reading. The error bars represent the standard deviation of repeated charge measurements within a single trial with the chamber reinserted between readings.

Fig. 7 shows average $R_{\mathrm{A} 12}^{\mathrm{A} 28}, R_{\mathrm{A} 12}^{\mathrm{IC} 69}$, and $R_{\mathrm{IC69}}^{\mathrm{A} 28}$ values for multiple trials normalized to the initial baseline ratio. The error bars represent the propagated Type A uncertainty from the standard deviation of the repeated charge measurements within a single trial.

\subsubsection{Linear accelerator measurements}

The total measurement time including setup needed to perform ten measurements with all three chambers for a given measurement trial at a single field size was approximately $13 \mathrm{~min}$. Fig. 8 shows individual chamber readings plotted over time. These readings shown were taken with all three chambers in place in the phantom. The error bars represent the standard deviation of repeated charge measurements for a single trial.

Eq. (2) was used to calculate charge ratios for the three chambers used in the accelerator beam. Fig. 9 shows average $R_{\mathrm{A} 12}^{\mathrm{A} 28}, R_{\mathrm{A} 12}^{\mathrm{IC} 69}$, and $R_{\mathrm{IC} 69}^{\mathrm{A} 28}$ values for multiple trials normalized to the initial baseline ratio in the 6 MV beam. For each trial shown, the error bars represent propagated Type A uncertainty for the measurements in a given trial.
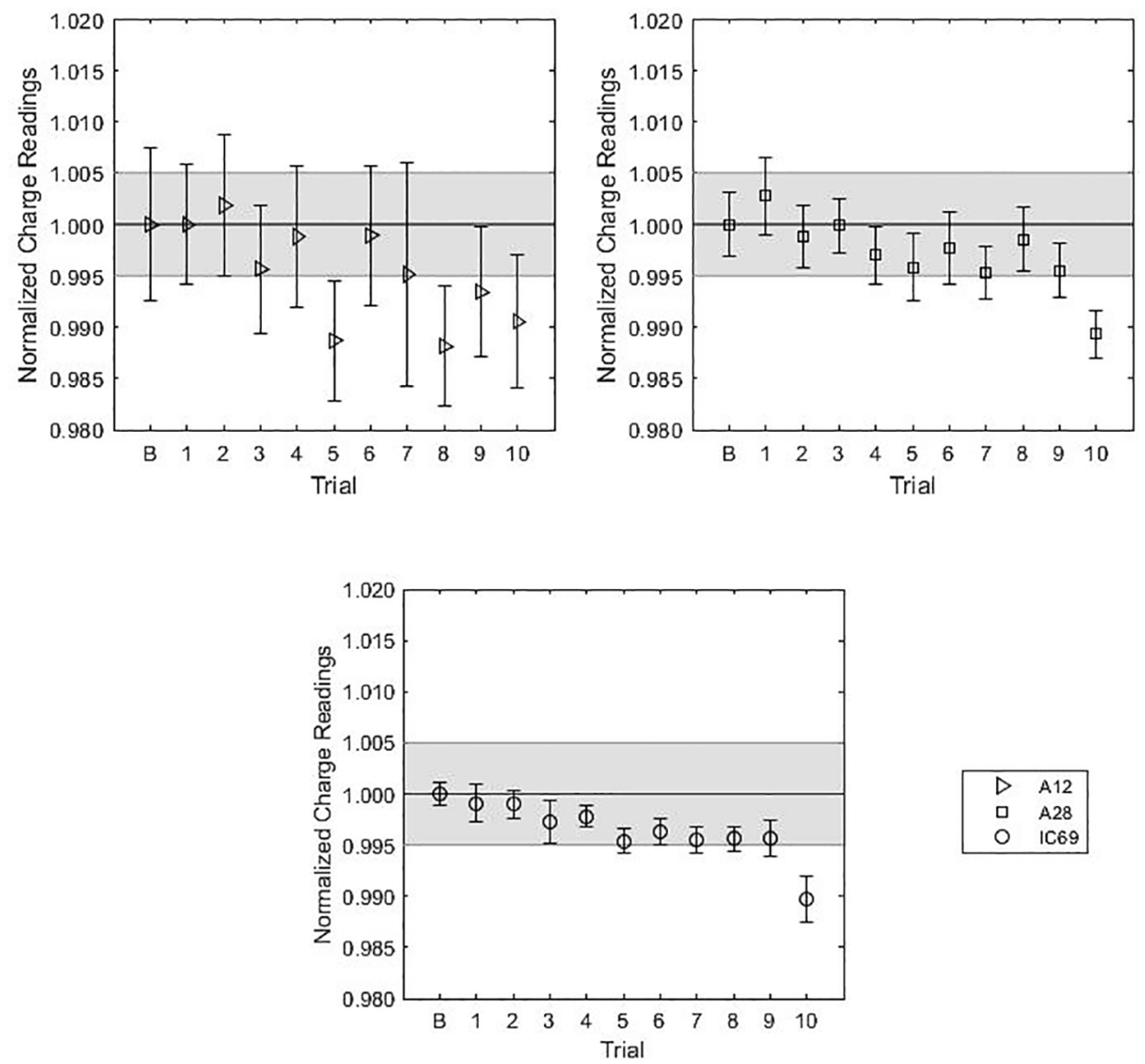

Fig. 6. Normalized charge readings in the ${ }^{90} \mathrm{Sr}$ check source for the A12, A28, and IC69 chambers. The highlighted region indicates a $\pm 0.5 \%$ difference from the initial reference value, which is denoted as Trial B. The error bars indicate the standard deviation of the repeated measurements in a given trial. 

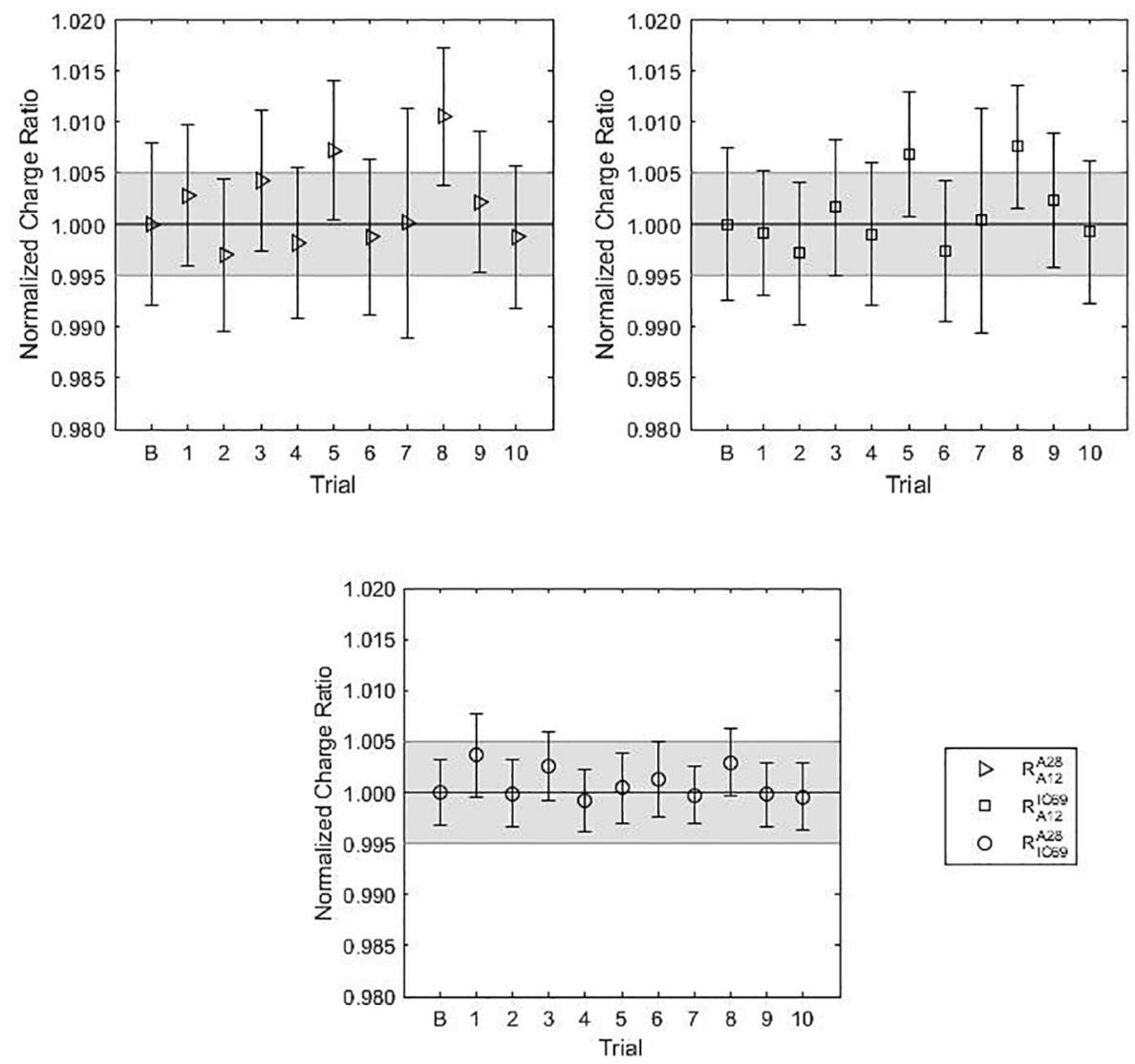

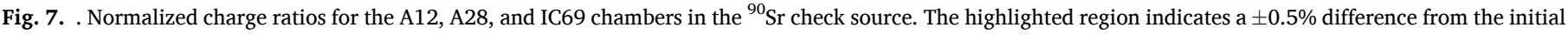
reference value, which is denoted as Trial $\mathrm{B}$. The error bars indicate the standard deviation of the repeated measurements in a given trial.

The standard deviation of the mean and the maximum difference from the baseline measurement for a given metric was used to assess the precision of the data. These values are listed in Table 1 for both the ${ }^{90} \mathrm{Sr}$ and linear accelerator measurements.

\subsubsection{Uncertainty}

The uncertainty budget for the ${ }^{90} \mathrm{Sr}$ measurements for individual chambers are given in Table 2. The Type A uncertainty listed for the charge is the maximum standard deviation observed across all three chambers for a single measurement trial. Included in this value is any influence from the positioning and rotational dependence chambers had within the source. As the A12 chamber had higher uncertainties than the A28 or IC69 chambers, this value is taken to be conservative. The leakage current was on the order of $1 \mathrm{fA}$ for all trials. The air density uncertainty comes from $\mathrm{a} \pm 1{ }^{\circ} \mathrm{C}$ and $\pm 1 \mathrm{kPa}$ uncertainty in the temperature and pressure measurements. The remaining terms in the uncertainty budget are determined from the UWADCL.

Table 3 gives the uncertainty budget for the linear accelerator measurements for individual chambers. The Type A uncertainty listed for the charge is the maximum value from a single measurement trial. The leakage current was on the order of $1 \mathrm{fA}$ for all trials. The air density uncertainty comes from $\mathrm{a} \pm 1{ }^{\circ} \mathrm{C}$ and $\pm 1 \mathrm{kPa}$ uncertainty in the temperature and pressure measurements. As temperature varied within each insert, this uncertainty was considered for all measurements. Additionally, the positioning uncertainty considers both positioning of the phantom within the beam as well as positioning of the chambers within the phantom, accounting for minimal effects from rotation of the chamber as well. The remaining terms in the uncertainty budget were previously determined at the UWADCL. The uncertainty for the ${ }^{60} \mathrm{Co}$ measurements includes similar values and yields an uncertainty of $0.41 \%$ at the $\mathrm{k}=2$ level.

\section{Discussion}

Measurements made in ${ }^{60} \mathrm{Co}$ indicate this method is a precise, stable means of tracking ionization chamber stability over time. It should be noted that the 2571 chamber had a lower response with respect to both the A12 and N30001G chambers. The overall spread of the data over time was comparable to the linear accelerator measurements, indicating that megavoltage beams are appropriate for these types of checks.

From the scanning data presented in Fig. 4, the chamber response was found to vary significantly with insertion depth in the ${ }^{90} \mathrm{Sr}$ check source. This result highlights the need for repeatable chamber positioning in the cavity, as small differences within a region of sharp dose falloff would lead to notable changes in chamber response. These changes would adversely affect the accuracy of chamber stability tests. While the A28 and IC69 chambers were placed reproducibly with the thimble tip resting on the bottom of the check source cavity, the A12 thimble tip was only able to reach a distance of about $1 \mathrm{~cm}$ from the well bottom. This resulted in less precise positioning for the A12 chamber within the dose gradient, leading to a larger standard deviation for charge measurements while using this chamber. This lack of precision indicates a limitation of the check source method.

Additionally, the chamber response was found to vary with rotation angle with respect to the ${ }^{90} \mathrm{Sr}$ source. The IC69 chamber proved to be the least sensitive to rotational variation with respect to the other two chambers. The angular dependence likely comes about from the slight tilt of the chamber within the check source cavity, and the response was found to be repeatable for multiple reinsertions of the chamber at each angle. When performing stability checks with a ${ }^{90} \mathrm{Sr}$ check source in the clinic, it is recommended that the chamber orientation be maintained between measurements to reduce unnecessary variation from rotational effects. 

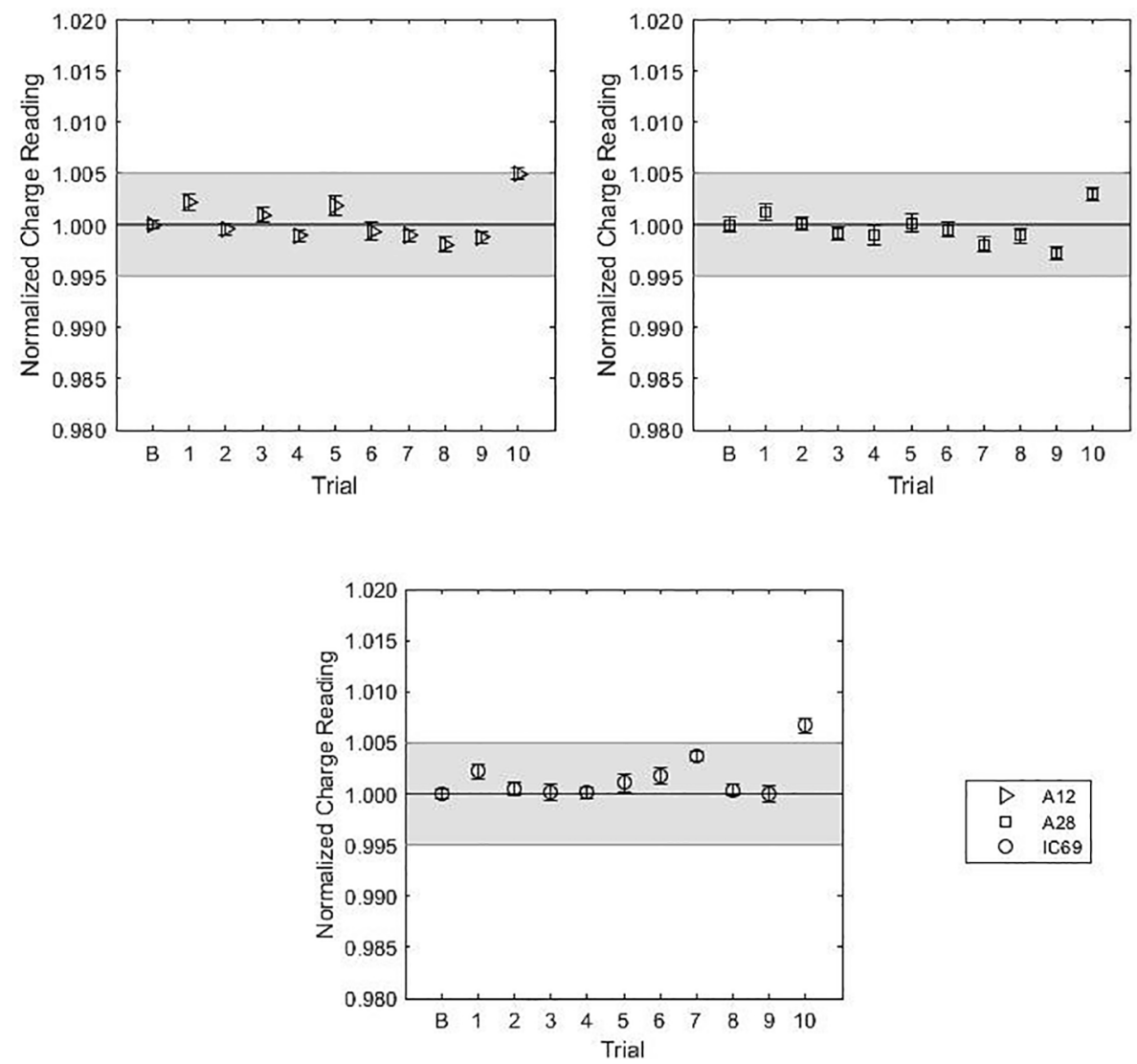

Fig. 8. Normalized charge readings in the $20 \times 20 \mathrm{~cm}^{2}$ field for the A12, A28, and IC69 chambers in the 6 MV beam. The highlighted region indicates a $\pm 0.5 \%$ difference from the initial reference value, which is denoted as Trial B. The error bars indicate the standard deviation of the repeated measurements in a given trial.

Fig. 6 presents normalized charge readings for the three chambers using the ${ }^{90} \mathrm{Sr}$ check source. Fig. 7 shows the normalized charge ratios for several chamber pairs. The downward drift for data points in Fig. 6 alone would suggest that there is a potential change in the chamber behavior and would be cause for concern about the stability of the chamber. However, no such trend is indicated for the ratios plotted in Fig. 7.

The larger standard deviations for $R_{\mathrm{A} 12}^{\mathrm{A} 28}$ and $R_{\mathrm{A} 12}^{\mathrm{IC} 69}$ within the ${ }^{90} \mathrm{Sr}$ check source can mainly be attributed to the larger standard deviations observed for the A12 charge measurements. The data for $R_{\mathrm{IC69}}^{\mathrm{A} 28}$ showed less spread between trials than the other ratios in the ${ }^{90} \mathrm{Sr}$ check source, but propagated Type A uncertainties for individual trials were still greater than those for the external beam measurements. On average, less variability was observed with the linear accelerator measurements than the check source measurements. Additionally, the linear accelerator measurements took approximately half the time of the ${ }^{90} \mathrm{Sr}$ measurements, indicating it is a more time efficient method.

Results from both the linear accelerator and ${ }^{90} \mathrm{Sr}$ check source highlight the benefit of comparing more than one chamber when monitoring stability. The ratios proved to be less sensitive to variations in the source of radiation, therefore making it easier to determine any clear changes in the chambers. Thus, the use of ratios with either redundant methods or inter-comparing single charge readings can provide an advantage in the clinical setting for a more precise monitoring of chamber stability.
The values observed for the spread of the data of individual chambers as well as the standard deviations of the individual trials were all within the uncertainty budgets in Tables 2 and 3 . The positioning of the chambers relative to the source played the largest contribution to the uncertainty in the measurements. Variation of the individual charge readings, with the exception of the IC69 in the external beam was within the quoted uncertainty for the respective measurement method.

\section{Conclusions}

This work highlights the importance of comparing more than one chamber when assessing chamber stability to eliminate influence from variability in radiation sources. Additionally, results show the linear accelerator provides a more precise and efficient method for evaluating the stability of ionization chambers due to less positioning uncertainty. The external beam measurements are also more compatible with a variety of chambers, while the check source is limited by the size of the opening. Overall, this work provides a comparison to allow the clinician to make an informed decision on their methods for evaluation of chamber stability.

\section{Disclosure}

Larry DeWerd has a partial interest in Standard Imaging, Inc. that manufactured the phantom purchased for this work to carry out linear accelerator measurements. 

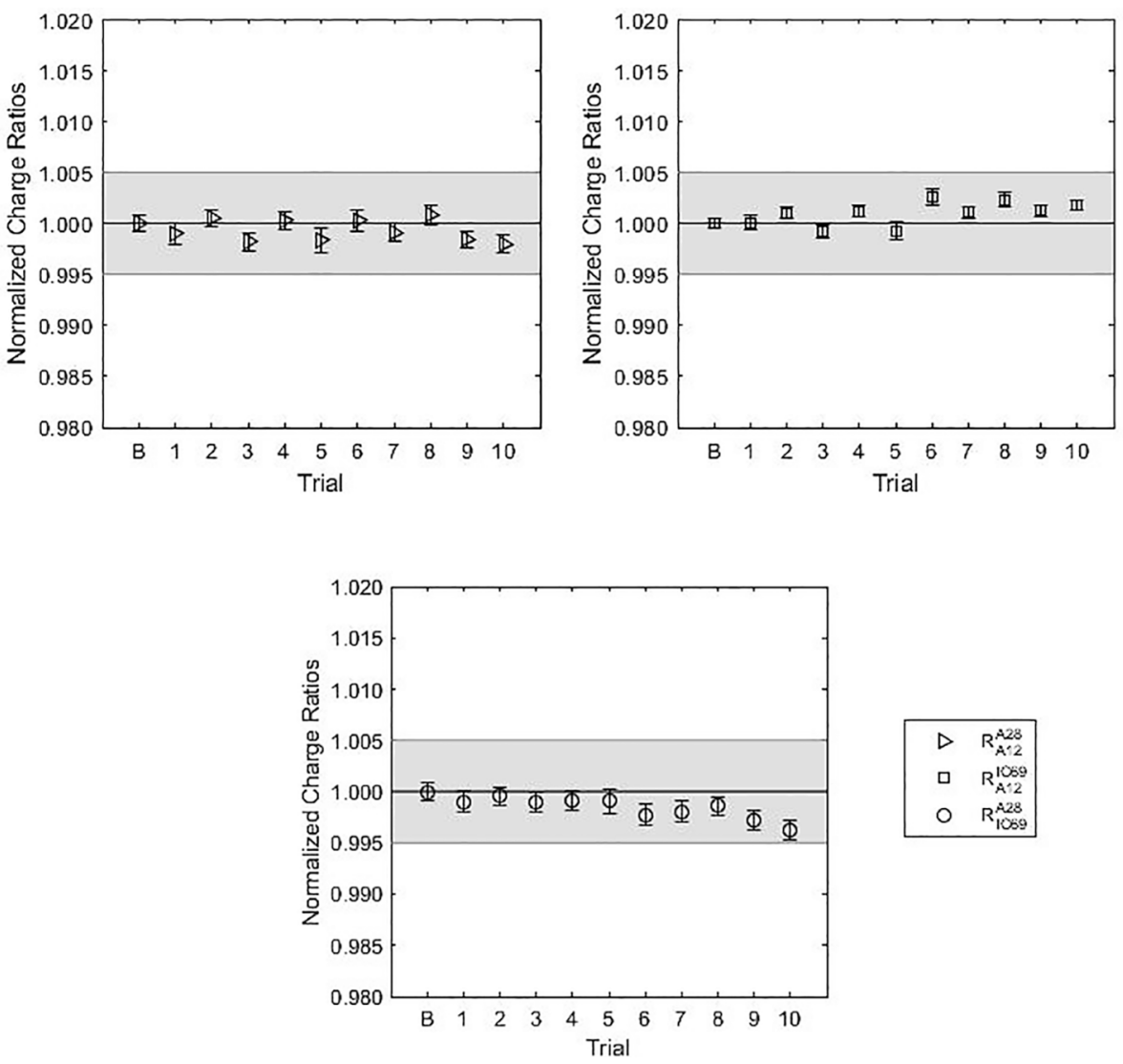

Fig. 9. Normalized charge ratios in the $20 \times 20 \mathrm{~cm}^{2}$ field for the A12, A28, and IC69 chambers in the $6 \mathrm{MV}$ beam. The highlighted region indicates a $\pm 0.5 \%$ difference from the initial reference value, which is denoted as Trial B. The error bars indicate the standard deviation of the repeated measurements in a given trial.

Table 1

Standard deviations of the means and maximum difference in measurements for both individual chambers and charge ratios in the ${ }^{90} \mathrm{Sr}$ check source and linear accelerator.

\begin{tabular}{llllll}
\hline & \multicolumn{2}{c}{${ }^{90} \mathbf{S r}$} & & \multicolumn{2}{c}{ Linear Accelerator } \\
\cline { 2 - 3 } \cline { 5 - 6 } $\begin{array}{l}\text { Standard } \\
\begin{array}{l}\text { Deviation of } \\
\text { the Mean }\end{array}\end{array}$ & $\begin{array}{l}\text { Maximum } \\
\text { difference from } \\
\text { baseline }\end{array}$ & & $\begin{array}{l}\text { Standard } \\
\text { Deviation of } \\
\text { the Mean }\end{array}$ & $\begin{array}{l}\text { Maximum } \\
\text { difference from } \\
\text { baseline }\end{array}$ \\
\hline $\mathrm{A} 12$ & $\pm 0.48 \%$ & $1.18 \%$ & $\pm 0.19 \%$ & $0.19 \%$ \\
$\mathrm{~A} 28$ & $\pm 0.35 \%$ & $1.07 \%$ & & $\pm 0.15 \%$ & $0.28 \%$ \\
$\mathrm{IC69}$ & $\pm 0.28 \%$ & $1.02 \%$ & $\pm 0.20 \%$ & $0.77 \%$ \\
$R_{\mathrm{A} 12}^{\mathrm{A} 28}$ & $\pm 0.40 \%$ & $1.05 \%$ & $\pm 0.10 \%$ & $0.20 \%$ \\
$R_{\mathrm{A} 12}^{\mathrm{II} 69}$ & $\pm 0.33 \%$ & $0.75 \%$ & $\pm 0.11 \%$ & $0.28 \%$ \\
$R_{\mathrm{IC69}}^{\mathrm{A} 28}$ & $\pm 0.15 \%$ & $0.36 \%$ & $\pm 0.11 \%$ & $0.48 \%$ \\
\hline
\end{tabular}

Table 3

Uncertainty budget for individual chamber measurements using the linear accelerator. Similar uncertainty can be assumed for the ratios.

\begin{tabular}{lll}
\hline Quantity & Type A & Type B \\
\hline Charge & $0.10 \%$ & $0.10 \%$ \\
Leakage Current & & $0.01 \%$ \\
Air Density & & $0.10 \%$ \\
Positioning & & $0.10 \%$ \\
Monitor Chamber & & $0.10 \%$ \\
Beam Uniformity & & $0.06 \%$ \\
Electrometer Calibration & & $0.11 \%$ \\
Combined Uncertainty & $0.10 \%$ & $0.21 \%$ \\
Standard Total Uncerainty (k=1) & & $\mathbf{0 . 2 6 \%}$ \\
Expanded Uncertainty $(\mathbf{k}=\mathbf{2})$ & $\mathbf{0 . 5 2 \%}$ & \\
\hline
\end{tabular}

Table 2

Uncertainty budget for the individual chamber measurements using the ${ }^{90} \mathrm{Sr}$ check source.

\begin{tabular}{lll}
\hline Quantity & Type A & Type B \\
\hline Charge & $0.99 \%$ & $0.10 \%$ \\
Leakage Current & & $0.01 \%$ \\
Air Density & & $0.10 \%$ \\
Orientation of Chamber & & $0.50 \%$ \\
Decay of Source & & $0.01 \%$ \\
Electrometer Calibration & $0.99 \%$ & $0.11 \%$ \\
Combined Uncertainty & & $0.53 \%$ \\
Standard Total Uncerainty $(\mathbf{k}=\mathbf{1})$ & & $\mathbf{1 . 1 2 \%}$ \\
Expanded Uncertainty $(\mathbf{k}=\mathbf{2})$ & & $\mathbf{2 . 2 5 \%}$ \\
\hline
\end{tabular}




\section{Acknowledgements}

The authors would like to thank the staff and customers of the UWADCL, whose continued patronage helps fund student research. We thank Stephen Bazan for the cobalt data. This research did not receive any specific grant from funding agencies in the public, commercial, or not-for-profit sectors.

\section{References}

[1] ICRU. Clinical Dosimetry: ICRU Report 10d. Washington DC: International Commission on Radiological Units and Measurements; 1962.

[2] ICRU. Radiation Dosimetry: X-rays generated at potentials of 5 to $150 \mathrm{KV}$. Washington, DC: International Commission on Radiation Units and Measurements: 1970.

[3] Almond PR, Biggs PJ, Coursey BM, Hanson WF, Huq MS, Nath R, et al. AAPM's TG 51 protocol for clinical reference dosimetry of high -energy photon and electron beams. Med Phys 1999;26(9):1847-70. https://doi.org/10.1118/1.598691.
[4] McEwen M, DeWerd L, Ibbott G, Followill D, Rogers DW, Seltzer S, et al. Addendum to the AAPM's TG-51 protocol for clinical reference dosimetry of highenergy photon beams. Med Phys 2014;41(4):041051. https://doi.org/10.1118/ 1.4866223 .

[5] American College of Radiology. ACR-AAPM technical standard for the performance of radiation oncology physics for external beam therapy; 2015.

[6] IEC. Medical electrical equipment-Dosimeters with ionization chambers as used in radiotherapy. Geneva, Switzerland: International Electrotechnical commission; 2011.

[7] Sidhu NPS, Rouvas A, Cadman P. Use of a radioactive check device for redundancy check of ionization chambers. J Appl Clin Med Phys 2000;1(4):148-52. https:// doi.org/10.1120/jacmp.v1i4.2636.

[8] McEwen MR, Taank J. Examining the influence of humidty on reference ionization chamber performance. Med Phys 2017;44(2):694-702. https://doi.org/10.1002 /mp.12057.

[9] Standards for Protection Against Radiation, 10 C.F.R. S 20; 1991.

[10] Rozenfeld M, Jette D. Quality assurance of radiation dosage: usefulness of redundancy. Radiology 1984;150(1):214-44. https://doi.org/10.1148/radiology. 150.1.6689767.

[11] Licenses and Radiation Safety Requirements for Irradiators, 10 C.F.R. S 36; 1983. 